\title{
Quaternionic Analysis and the Schrödinger Model for the Minimal Representation of $O(3,3)$
}

\author{
Igor Frenkel and Matvei Libine
}

November 17, 2018

\begin{abstract}
In the series of papers FL1, FL2 we approach quaternionic analysis from the point of view of representation theory of the conformal group $S L\left(2, \mathbb{H}_{\mathbb{C}}\right) \simeq S L(4, \mathbb{C})$ and its real forms. This approach has proven very fruitful and pushed further the parallel with complex analysis and develop a rich theory. In [FL2] we study the counterparts of Cauchy-Fueter and Poisson formulas on the spaces of split quaternions $\mathbb{H}_{\mathbb{R}}$ and Minkowski space $\mathbb{M}$ and show that they solve the problem of separation of the discrete and continuous series on $S L(2, \mathbb{R})$ and the imaginary Lobachevski space $S L(2, \mathbb{C}) / S L(2, \mathbb{R})$. In particular, we introduce an operator $\mathrm{Pl}_{R}$, compute its effect on the discrete and continuous series components of the space of functions $\mathcal{H}\left(\mathbb{H}_{\mathbb{R}}^{+}\right)$and obtain a surprising formula for the Plancherel measure of $S L(2, \mathbb{R})$. The proof is based on a transition to the Minkowski space $\mathbb{M}$ and some pretty lengthy computations. In this paper we introduce an operator $\frac{d}{d R} \mathrm{Pl}_{R}$ on $\mathcal{H}\left(\mathbb{H}_{\mathbb{R}}^{+}\right)$and show that its effect on the discrete and continuous series components can be easily computed using the Schrödinger model for the minimal representation of $O(p, q)$ (with $p=q=3$ ) and the results of Kobayashi-Mano from [KM], particularly their computation of the integral expression for the operator $\mathcal{F}_{C}$. This provides an independent verification of the coefficients involved in the formula for $\mathrm{Pl}_{R}$. This paper once again demonstrates a close connection between quaternionic analysis and representation theory of various $O(p, q)$ 's.
\end{abstract}

\section{Introduction}

In the series of papers [FL1, FL2] we approach quaternionic analysis from the point of view of representation theory of the conformal group $S L\left(2, \mathbb{H}_{\mathbb{C}}\right) \simeq S L(4, \mathbb{C})$ and its real forms such as $S L(2, \mathbb{H}) \approx S O(5,1), S U(2,2) \approx S O(4,2), S L(4, \mathbb{R}) \approx S O(3,3)$. This approach has proven very fruitful and pushed further the parallel with complex analysis and develop a rich theory. In FL2 we study the counterparts of Cauchy-Fueter and Poisson formulas on the spaces of split quaternions $\mathbb{H}_{\mathbb{R}}$ and Minkowski space $\mathbb{M}$ and show that they solve the problem of separation of the discrete and continuous series on $S L(2, \mathbb{R})$ and the imaginary Lobachevski space $S L(2, \mathbb{C}) / S L(2, \mathbb{R})$. The continuous series component on $S L(2, \mathbb{R})$ gives rise to the minimal representation of the conformal group $S L(4, \mathbb{R})$. We also obtain a surprising formula for the Plancherel measure of $S L(2, \mathbb{R})$ in terms of the Poisson integral on the split quaternions $\mathbb{H}_{\mathbb{R}}$ (Theorem 94). For convenience we restate it here.

Theorem 1 The operator defined on the space of functions $\mathcal{H}\left(\mathbb{H}_{\mathbb{R}}^{+}\right)$

$$
\varphi(X) \mapsto\left(\mathrm{Pl}_{R} \varphi\right)(W)=\lim _{\varepsilon \rightarrow 0^{+}} \frac{1}{\pi^{2}} \int_{X \in H_{R}}\left(\frac{1}{N(X-W)+i \varepsilon}-\frac{1}{N(X-W)-i \varepsilon}\right) \varphi(X) \frac{d S}{\|X\|}
$$


annihilates the discrete series component of $\mathcal{H}\left(\mathbb{H}_{\mathbb{R}}^{+}\right)$. If $\operatorname{Re} l=-\frac{1}{2}$ and $W \in \mathbb{H}_{\mathbb{R}}^{+}$,

$$
\left(\mathrm{Pl}_{R} t_{n \underline{m}}^{l}\right)(W)=\left(1+R^{4 \operatorname{Im} l} \cdot N(W)^{-2 l-1}\right) \cdot t_{n \underline{m}}^{l}(W) \cdot \begin{cases}\frac{\operatorname{coth}(\pi \operatorname{Im} l)}{\operatorname{Im} l} & \text { if } m, n \in \mathbb{Z} ; \\ \frac{\tanh (\pi \operatorname{Im} l)}{\operatorname{Im} l} & \text { if } m, n \in \mathbb{Z}+\frac{1}{2} .\end{cases}
$$

Of course, strictly speaking, the restrictions to $S U(1,1)$ of the matrix coefficient functions of the continuous series representations $t_{n \underline{m}}^{l}(X), l=-1 / 2+i \lambda$ with $\lambda \in \mathbb{R}$, do not belong to $L^{2}(S U(1,1))$, so we need to work with wave packets, as explained in Remark 93 in [FL2].

It is relatively easy to see that the operator $\mathrm{Pl}_{R}$ annihilates the discrete series and that $\mathrm{Pl}_{R}$ is $S U(1,1) \times S U(1,1)$-equivariant, but it is much harder to determine its effect on the continuous series. The proof given in FL2 is based on a transition to the Minkowski space $\mathbb{M}$ and some pretty lengthy computations. It is highly desirable to find a more simple or at the very least a less computational proof.

In this paper we give an elementary proof of a slightly weaker result (Theorem 2). This proof uses the setting of the Schrödinger model for the minimal representation 1 of $O(p, q)$ (with $p=q=3$ ) and the results of Kobayashi-Mano from [KM], particularly their computation of the integral expression for the operator $\mathcal{F}_{C}$. This provides an independent verification of the coefficients involved in Theorem 1. It is interesting to note that our argument can be reversed and, assuming Theorem 2, one can obtain the integral expression for the operator $\mathcal{F}_{C}$ when $p=q=3$. The Minkowski space $\mathbb{M}$ is also implicitly present in this article. We are essentially computing the ratio of two integral kernels from $[\mathrm{KM}]$ - one for $\mathbb{H}_{\mathbb{R}}$ and the other for $\mathbb{M}$.

In addition to proving Theorem 2, we find all $K$-types of the minimal representation of $S O(3,3)$ (in the Schrödinger model realization). Some - but not all - $K$-types of the minimal representations $V^{p, q}$ of various $O(p, q)$ 's were found in $[\mathrm{KM}$. Kobayashi and Mano called the $K$-types they found the "skeleton" of the minimal representation in the sense that they contain at least one non-zero vector from each $K$-irreducible component of $V^{p, q}$. Moreover, as was pointed out by the referee, more $K$-types were found by Hilgert, Kobayashi, Mano and Möllers in [HKMM]. More precisely, they fix a certain subgroup $K^{\prime}$ of $K=O(p) \times O(q)$ isomorphic to $O(p-1) \times O(q-1)$; then Corollary 8.2 in [HKMM] describes all $K$-types of $V^{p, q}$ that are fixed by $K^{\prime}$ and asserts that each $K$-irreducible component of $V^{p, q}$ contains a unique (up to scaling) non-zero $K^{\prime}$-fixed vector.

Our paper once again demonstrates a close connection between quaternionic analysis and representation theory of various $O(p, q)$ 's.

The first author was supported by the NSF grants DMS-0457444 and DMS-1001633; the second author was supported by the NSF grant DMS-0904612.

\section{Statement of the Main Result}

Let us recall the $\delta$-functions on the cone $C=\left\{X \in \mathbb{H}_{\mathbb{R}} ; N(X)=0\right\}$ and the hyperboloids $H_{R}=\left\{X \in \mathbb{H}_{\mathbb{R}} ; N(X)=R^{2}\right\}$, denoted by $\delta(C)$ and $\delta\left(H_{R}\right)$ respectively,

$$
\begin{gathered}
\delta(C)=\frac{1}{2 \pi i}\left(\frac{1}{N(X)-i 0}-\frac{1}{N(X)+i 0}\right): \quad \psi \mapsto \frac{1}{2} \int_{X \in C} \psi(X) \frac{d S}{\|X\|}, \\
\delta\left(H_{R}\right)=\frac{1}{2 \pi i}\left(\frac{1}{N(X)-R^{2}-i 0}-\frac{1}{N(X)-R^{2}+i 0}\right): \quad \psi \mapsto \frac{1}{2} \int_{X \in H_{R}} \psi(X) \frac{d S}{\|X\|} .
\end{gathered}
$$

\footnotetext{
${ }^{1}$ Strictly speaking, $S O(3,3)$ does not have a minimal representation, so by "minimal representation of $S O(3,3)$ " we mean the representation $\left(\varpi^{p, q}, V^{p, q}\right)$ of $O(p, q)$ in the notations of [KØ], [KM] for $p=q=3$. When $p+q$ is an even number greater than or equal 8 , one gets a genuine minimal representation.
} 
Using these distributions we can formally rewrite the operator $\mathrm{Pl}_{R}$ introduced in [FL2] as

$$
\mathrm{Pl}_{R}: \quad \varphi \mapsto \frac{4}{\pi i}\left(\varphi \cdot \delta\left(H_{R}\right)\right) * \delta(C),
$$

where $*$ denotes the convolution and either $\delta(C)$ or $\delta\left(H_{R}\right)$ should be replaced with

$$
\begin{aligned}
\delta(C)(\psi) & =\lim _{\varepsilon \rightarrow 0^{+}} \frac{1}{2 \pi i} \int_{X \in \mathbb{H}_{\mathbb{R}}}\left(\frac{1}{N(X)-i \varepsilon}-\frac{1}{N(X)+i \varepsilon}\right) \psi(X) d V \quad \text { or } \\
\delta\left(H_{R}\right)(\psi) & =\lim _{\varepsilon \rightarrow 0^{+}} \frac{1}{2 \pi i} \int_{X \in \mathbb{H}_{\mathbb{R}}}\left(\frac{1}{N(X)-R^{2}-i \varepsilon}-\frac{1}{N(X)-R^{2}+i \varepsilon}\right) \psi(X) d V,
\end{aligned}
$$

with $d V=d x^{0} \wedge d x^{1} \wedge d x^{2} \wedge d x^{3}$. Thus we can rewrite $\mathrm{Pl}_{R}$ as

$\left(\mathrm{Pl}_{R} \varphi\right)(W)=\lim _{\varepsilon \rightarrow 0^{+}} \frac{1}{\pi^{2}} \int_{X \in C}\left(\frac{1}{N(W-X)-R^{2}+i \varepsilon}-\frac{1}{N(W-X)-R^{2}-i \varepsilon}\right) \varphi(W-X) \frac{d S}{\|X\|}$.

Differentiating with respect to $R^{2}$ we obtain the following result:

Theorem 2 The operator

$$
\begin{aligned}
& \varphi(X) \mapsto\left(\mathrm{Pl}_{R}^{\prime} \varphi\right)(W) \\
& =\lim _{\varepsilon \rightarrow 0^{+}} \frac{1}{2 \pi^{2}} \int_{X \in C}\left(\frac{1}{\left(N(W-X)-R^{2}+i \varepsilon\right)^{2}}-\frac{1}{\left(N(W-X)-R^{2}-i \varepsilon\right)^{2}}\right) \varphi(W-X) \frac{d S}{\|X\|}
\end{aligned}
$$

annihilates the discrete series component of $\mathcal{H}\left(\mathbb{H}_{\mathbb{R}}^{+}\right)$. If $\operatorname{Re} l=-\frac{1}{2}$ and $W \in \mathbb{H}_{\mathbb{R}}^{+}$,

$$
\left(\mathrm{Pl}_{R}^{\prime} t_{n \underline{m}}^{l}\right)(W)=R^{4 l} \cdot N(W)^{-2 l-1} \cdot t_{n \underline{m}}^{l}(W) \cdot \begin{cases}\operatorname{coth}(\pi \operatorname{Im} l) & \text { if } m, n \in \mathbb{Z} \\ \tanh (\pi \operatorname{Im} l) & \text { if } m, n \in \mathbb{Z}+\frac{1}{2} .\end{cases}
$$

The point of introducing the operator $\mathrm{Pl}_{R}^{\prime}$ is that its Fourier transform is particularly easy to compute.

\section{Schrödinger Model for the Minimal Representation of $O(3,3)$}

Let $w_{0}=\left(\begin{array}{cc}I_{p} & 0 \\ 0 & -I_{q}\end{array}\right) \in O(p, q)$, where $I_{p}$ and $I_{q}$ denote the $p \times p$ and $q \times q$ identity matrices. One of the main results of $[\mathrm{KM}]$ is finding an explicit expression for the action of $w_{0}$ on the minimal representation of $O(p, q)$ realized in the Schrödinger model $\left(\pi, L^{2}\left(C^{*}\right)\right)$. This explicit expression is then used to prove unitarity of the minimal representation. In this paper we are interested in the case $p=q=3$. Let $w_{0}^{\prime} \in O(3,3)$ be the diagonal matrix with diagonal entries $(-1,1,1,1,1,1)$. The action of $O(3,3)$ on $\mathbb{R}^{2,2}$ is described in Subsection 2.8 of Part III of [KØ], and it is easy to see that the element $w_{0}^{\prime}$ acts on $\mathbb{R}^{2,2}$ by

$$
w_{0}^{\prime}: \quad\left(u^{\prime}, u^{\prime \prime}\right) \mapsto \frac{4}{\left|u^{\prime}\right|^{2}-\left|u^{\prime \prime}\right|^{2}}\left(u^{\prime}, u^{\prime \prime}\right)
$$

This action lifts to the solutions of the ultrahyperbolic wave equation:

$$
w_{0}^{\prime}: \quad \varphi\left(u^{\prime}, u^{\prime \prime}\right) \mapsto \frac{4}{\left|u^{\prime}\right|^{2}-\left|u^{\prime \prime}\right|^{2}} \cdot \varphi\left(\frac{4}{\left|u^{\prime}\right|^{2}-\left|u^{\prime \prime}\right|^{2}}\left(u^{\prime}, u^{\prime \prime}\right)\right) .
$$


We identify the split quaternions $\mathbb{H}_{\mathbb{R}}$ with $\mathbb{R}^{2,2}$ as follows:

$$
\begin{gathered}
\mathbb{H}_{\mathbb{R}}=\left\{X=x_{1} e_{0}+x_{3} \tilde{e}_{1}+x_{4} \tilde{e}_{2}+x_{2} e_{3}=\left(\begin{array}{cc}
x_{1}-i x_{2} & x_{3}+i x_{4} \\
x_{3}-i x_{4} & x_{1}+i x_{2}
\end{array}\right) ; x_{1}, x_{2}, x_{3}, x_{4} \in \mathbb{R}\right\}, \\
\mathbb{H}_{\mathbb{R}} \ni X \longleftrightarrow\left(u^{\prime}, u^{\prime \prime}\right) \in \mathbb{R}^{2,2}, \quad u^{\prime}=\left(x_{1}, x_{2}\right), \quad u^{\prime \prime}=\left(x_{3}, x_{4}\right),
\end{gathered}
$$

this way

$$
\square_{2,2}=\frac{\partial^{2}}{\partial x_{1}^{2}}+\frac{\partial^{2}}{\partial x_{2}^{2}}-\frac{\partial^{2}}{\partial x_{3}^{2}}-\frac{\partial^{2}}{\partial x_{4}^{2}} .
$$

Then $w_{0}^{\prime}$ corresponds to the operator on $\mathbb{H}_{\mathbb{R}}$ and the solutions of $\square_{2,2} \varphi=0$

$$
w_{0}^{\prime}: \quad X \mapsto \frac{4 X}{N(X)}, \quad \varphi(X) \mapsto \frac{4}{N(X)} \cdot \varphi\left(\frac{4 X}{N(X)}\right) .
$$

In particular, $w_{0}^{\prime}$ acts on the matrix coefficients $t_{n \underline{m}}^{l}$ 's of $S U(1,1)$ (see Subsection 2.5 in [FL2]) by

$$
w_{0}^{\prime}: \quad t_{n \underline{m}}^{l}(X) \mapsto 2^{4 l+2} \cdot N(X)^{-2 l-1} \cdot t_{n \underline{m}}^{l}(X) .
$$

As in $[\mathrm{KM}]$, we use the following normalization of the Fourier transform on $\mathbb{H}_{\mathbb{R}}$ :

$$
\hat{\psi}(\xi)=\frac{1}{(2 \pi)^{2}} \int_{X \in \mathbb{H}_{\mathbb{R}}} \psi(X) \cdot e^{i \xi \cdot X} d V
$$

where $\xi=\left(\xi_{1}, \xi_{2}, \xi_{3}, \xi_{4}\right) \in \mathbb{H}_{\mathbb{R}}^{*}$ - the dual of $\mathbb{H}_{\mathbb{R}}$ and $\xi \cdot X=\xi_{1} x_{1}+\xi_{2} x_{2}+\xi_{3} x_{3}+\xi_{4} x_{4}$. For $\xi, \xi^{\prime} \in \mathbb{H}_{\mathbb{R}}^{*}$, write

$$
\left\langle\xi, \xi^{\prime}\right\rangle=\xi_{1} \xi_{1}^{\prime}+\xi_{2} \xi_{2}^{\prime}-\xi_{3} \xi_{3}^{\prime}-\xi_{4} \xi_{4}^{\prime}, \quad \xi=\left(\xi_{1}, \xi_{2}, \xi_{3}, \xi_{4}\right), \quad \xi^{\prime}=\left(\xi_{1}^{\prime}, \xi_{2}^{\prime}, \xi_{3}^{\prime}, \xi_{4}^{\prime}\right) .
$$

Let $C^{*}$ denote the cone dual to $C$ :

$$
C^{*}=\left\{\xi \in \mathbb{H}_{\mathbb{R}}^{*} ;\langle\xi, \xi\rangle=\left(\xi_{1}\right)^{2}+\left(\xi_{2}\right)^{2}-\left(\xi_{3}\right)^{2}-\left(\xi_{4}\right)^{2}=0\right\},
$$

and let $\mathcal{F}_{C^{*}}, \mathcal{F}_{C^{*}}^{\prime}$ be integral operators on $L^{2}\left(C^{*}\right)$ :

$$
\begin{aligned}
& \left(\mathcal{F}_{C^{*}} f\right)(\xi)=-\frac{1}{\pi} \int_{\xi^{\prime} \in C^{*}} \Psi_{0}\left(\xi_{1} \xi_{1}^{\prime}+\xi_{2} \xi_{2}^{\prime}+\xi_{3} \xi_{3}^{\prime}+\xi_{4} \xi_{4}^{\prime}\right) \cdot f\left(\xi^{\prime}\right) \frac{d S}{\left\|\xi^{\prime}\right\|}, \\
& \left(\mathcal{F}_{C^{*}}^{\prime} f\right)(\xi)=-\frac{1}{\pi} \int_{\xi^{\prime} \in C^{*}} \Psi_{0}\left(-\left\langle\xi, \xi^{\prime}\right\rangle\right) \cdot f\left(\xi^{\prime}\right) \frac{d S}{\left\|\xi^{\prime}\right\|}, \quad f \in L^{2}\left(C^{*}\right),
\end{aligned}
$$

where

$$
\Psi_{0}(t)= \begin{cases}Y_{0}(2 \sqrt{2 t}), & t>0 \\ -\frac{2}{\pi} K_{0}(2 \sqrt{-2 t}), & t<0\end{cases}
$$

$Y_{0}$ is the Bessel function of the second kind, $K_{0}$ is the modified Bessel function of the second kind. As a special case of Theorem 5.1.1 in [KM], in the Schrödinger model $\left(\pi, L^{2}\left(C^{*}\right)\right)$ for the minimal representation of $O(3,3)$, the operator $\pi\left(w_{0}\right): L^{2}\left(C^{*}\right) \rightarrow L^{2}\left(C^{*}\right)$ is given by $\pi\left(w_{0}\right) f=\mathcal{F}_{C^{*}} f$. Since $w_{0}^{\prime}$ can be expressed as $w_{0}$ times a diagonal matrix with diagonal entries $(-1,1,1,-1,-1,-1)$ and $-I d \in O(3,3)$ acts on the minimal representation trivially, we immediately obtain that the operator $\pi\left(w_{0}^{\prime}\right): L^{2}\left(C^{*}\right) \rightarrow L^{2}\left(C^{*}\right)$ is given by $\pi\left(w_{0}^{\prime}\right) f=\mathcal{F}_{C^{*}}^{\prime} f$.

We finish this section by identifying $\mathfrak{s p}(2, \mathbb{R}) \simeq \mathfrak{s o}(3,2)$ sitting inside $\mathfrak{s l}\left(2, \mathbb{H}_{\mathbb{R}}\right) \simeq \mathfrak{s l}(4, \mathbb{R})$. Set $\varepsilon_{1}=\varepsilon_{2}=1, \varepsilon_{3}=\varepsilon_{4}=-1$ and let $\widehat{\operatorname{deg}}$ denote the degree operator plus identity:

$$
\widetilde{\operatorname{deg} f}=\xi_{1} \frac{\partial f}{\partial \xi_{1}}+\xi_{2} \frac{\partial f}{\partial \xi_{2}}+\xi_{3} \frac{\partial f}{\partial \xi_{3}}+\xi_{4} \frac{\partial f}{\partial \xi_{4}}+f .
$$


The differential operators acting on $L^{2}\left(C^{*}\right)$ as

$$
\begin{aligned}
X_{j k}= & \varepsilon_{j} \varepsilon_{k} \xi_{j} \frac{\partial}{\partial \xi_{k}}-\xi_{k} \frac{\partial}{\partial \xi_{j}}, \quad 1 \leq j<k \leq 4, \\
& i\left(\varepsilon_{j} P_{j}+4 \xi_{j}\right), \quad 1 \leq j \leq 4, \quad \text { where } P_{j}=\varepsilon_{j} \xi_{j} \square_{2,2}-\widetilde{\operatorname{deg}} \circ \frac{\partial}{\partial \xi_{j}},
\end{aligned}
$$

generate a Lie algebra isomorphic to $\mathfrak{s p}(2, \mathbb{R}) \simeq \mathfrak{s o}(3,2)$. We use Lemma 17 from [FL1] describing $\mathfrak{s l}\left(2, \mathbb{H}_{\mathbb{R}}\right)$ action on the space of harmonic functions (see also its corrected version at the beginning of Subsection 3.2 in [FL2]). By direct computation we can see that the elements $\left(\begin{array}{cc}A & 0 \\ 0 & D\end{array}\right)$ with $A, D \in \mathbb{H}_{\mathbb{R}}, A^{+}=-A, D^{+}=-D$ act by linear combinations of $X_{j k}$ which generate a Lie algebra isomorphic to $\mathfrak{s l}(2, \mathbb{R}) \times \mathfrak{s l}(2, \mathbb{R})$. Next we see that elements $\left(\begin{array}{ll}0 & B \\ 0 & 0\end{array}\right)$ correspond to linear combinations of multiplication operators $i \xi_{j}, 1 \leq j \leq 4$. More specifically, choosing $B$ equal $e_{0}, \tilde{e}_{1}, \tilde{e}_{2}$ and $e_{3}$ gives rise to multiplication by $i \xi_{1}, i \xi_{3}, i \xi_{4}$ and $i \xi_{2}$ respectively. Finally, by direct computation we get

$$
\begin{aligned}
2(X \partial X-X)=e_{0}\left(-N(X) \frac{\partial}{\partial x_{1}}\right. & \left.+2 x_{1} \widetilde{\operatorname{deg}}\right)+\tilde{e}_{1}\left(N(X) \frac{\partial}{\partial x_{3}}+2 x_{3} \widetilde{\operatorname{deg}}\right) \\
& +\tilde{e}_{2}\left(N(X) \frac{\partial}{\partial x_{4}}+2 x_{4} \widetilde{\operatorname{deg}}\right)+e_{3}\left(-N(X) \frac{\partial}{\partial x_{2}}+2 x_{2} \widetilde{\operatorname{deg}}\right)
\end{aligned}
$$

which implies that elements $\left(\begin{array}{cc}0 & 0 \\ C & 0\end{array}\right)$ correspond to linear combinations of operators

$$
i P_{j}(-1)=i \varepsilon_{j} \xi_{j} \square_{2,2}-i(2 \widetilde{\operatorname{deg}}+4) \circ \frac{\partial}{\partial \xi_{j}}, \quad 1 \leq j \leq 4
$$

More specifically, choosing $C$ equal $e_{0}, \tilde{e}_{1}, \tilde{e}_{2}$ and $e_{3}$ gives rise to operators $-i P_{1}(-1),-i P_{3}(-1)$, $-i P_{4}(-1)$ and $i P_{2}(-1)$ respectively. This implies that the Lie algebra generated by the differential operators $X_{j k}$ and $i\left(\varepsilon_{j} P_{j}+4 \xi_{j}\right)$ corresponds to

$$
\left\{\left(\begin{array}{cc}
A & 4 B \\
-B^{+} & D
\end{array}\right) ; A, B, D \in \mathbb{H}_{\mathbb{R}}, A^{+}=-A, D^{+}=-D\right\} \subset \mathfrak{s l}\left(2, \mathbb{H}_{\mathbb{R}}\right) .
$$

The " 4 " appears in the description of the Lie algebra because of the " 4 " in $i\left(\varepsilon_{j} P_{j}+4 \xi_{j}\right)$. The connected Lie subgroup of $G L\left(2, \mathbb{H}_{\mathbb{R}}\right)$ with the above Lie algebra preserves the hyperboloid $\left\{X \in \mathbb{H}_{\mathbb{R}} ; N(X)=-4\right\}$ (see Lemma 25 in [FL2]).

\section{$4 K$-finite Vectors of the Minimal Representation of $O(3,3)$}

In this section we find the $K$-finite vectors of the minimal representation of $O(3,3)$ realized in $L^{2}\left(C^{*}\right)$. This section is not needed for the proof of Theorem 2. Following [KM], we renormalize the K-Bessel functions as

$$
\tilde{K}_{n}(r)=2^{n} r^{-n} K_{n}(r), \quad n \in \mathbb{Z} .
$$

We have the following relation between K-Bessel functions:

$$
K_{n+1}(r)-K_{n-1}(r)=2 n r^{-1} K_{n}(r),
$$

which implies

$$
r^{2} \tilde{K}_{n+1}(2 r)=n \tilde{K}_{n}(2 r)+\tilde{K}_{n-1}(2 r) .
$$


The derivatives of K-Bessel functions satisfy the following relation:

$$
\left(-\frac{d}{r d r}\right)^{m}\left(r^{-n} K_{n}(r)\right)=r^{-n-m} K_{n+m}(r), \quad n \in \mathbb{Z},
$$

which implies

$$
\left(-\frac{2 d}{r d r}\right)^{m} \tilde{K}_{n}(r)=\tilde{K}_{n+m}(r), \quad \frac{d}{d r} \tilde{K}_{n}(2 r)=-2 r \tilde{K}_{n+1}(2 r), \quad n \in \mathbb{Z} .
$$

We identify $C^{*} \backslash\{0\}$ with $\mathbb{R}_{+} \times S^{1} \times S^{1}$ using coordinates

$$
\left(r, \theta_{1}, \theta_{2}\right) \longleftrightarrow \xi=r\left(\cos \theta_{1}, \sin \theta_{1}, \cos \theta_{2}, \sin \theta_{2}\right) \quad \in C^{*},
$$

which induces an isomorphism of Hilbert spaces

$$
L^{2}\left(C^{*}\right) \simeq L^{2}\left(\mathbb{R}_{+}, \frac{r}{2} d r\right) \hat{\otimes} L^{2}\left(S^{1}\right) \hat{\otimes} L^{2}\left(S^{1}\right) .
$$

Consider the following functions on the cone $C^{*}$ :

$$
\tilde{K}_{n}(2 r)\left(\xi_{1} \pm i \xi_{2}\right)^{l}\left(\xi_{3} \pm i \xi_{4}\right)^{k}=r^{l+k} \tilde{K}_{n}(2 r) e^{ \pm i l \theta_{1}} e^{ \pm i k \theta_{2}}
$$

$n \in \mathbb{Z}, k, l=0,1,2, \ldots$. Clearly, they are $S O(2) \times S O(2)$-finite.

The action of $\mathfrak{s l}(4, \mathbb{R})$ is generated by multiplication by $\xi_{i}, 1 \leq i \leq 4$, and differential operators $P_{j}=\varepsilon_{j} \xi_{j} \square_{2,2}-2 \widetilde{\operatorname{deg}} \circ \frac{\partial}{\partial \xi_{j}}, 1 \leq j \leq 4$. Hence it is important to find actions of these operators. The multiplication operators are easy. For example, using (4):

$$
\begin{aligned}
2 \xi_{1} \tilde{K}_{n}(2 r)\left(\xi_{1} \pm i \xi_{2}\right)^{l} & =\tilde{K}_{n}(2 r)\left(\xi_{1} \pm i \xi_{2}\right)^{l+1}+r^{2} \tilde{K}_{n}(2 r)\left(\xi_{1} \pm i \xi_{2}\right)^{l-1} \\
& =\tilde{K}_{n}(2 r)\left(\xi_{1} \pm i \xi_{2}\right)^{l+1}+\left((n-1) \tilde{K}_{n-1}(2 r)+\tilde{K}_{n-2}(2 r)\right)\left(\xi_{1} \pm i \xi_{2}\right)^{l-1} ; \\
2 i \xi_{2} \tilde{K}_{n}(2 r)\left(\xi_{1} \pm i \xi_{2}\right)^{l} & = \pm \tilde{K}_{n}(2 r)\left(\xi_{1} \pm i \xi_{2}\right)^{l+1} \mp r^{2} \tilde{K}_{n}(2 r)\left(\xi_{1} \pm i \xi_{2}\right)^{l-1} \\
& = \pm \tilde{K}_{n}(2 r)\left(\xi_{1} \pm i \xi_{2}\right)^{l+1} \mp\left((n-1) \tilde{K}_{n-1}(2 r)+\tilde{K}_{n-2}(2 r)\right)\left(\xi_{1} \pm i \xi_{2}\right)^{l-1} .
\end{aligned}
$$

To find the action of $P_{j}$ 's we observe that in bipolar coordinates

$$
\square_{2,2}=\frac{\partial^{2}}{\partial r_{1}^{2}}+\frac{1}{r_{1}} \frac{\partial}{\partial r_{1}}+\frac{1}{r_{1}^{2}} \frac{\partial^{2}}{\partial \theta_{1}^{2}}-\frac{\partial^{2}}{\partial r_{2}^{2}}-\frac{1}{r_{2}} \frac{\partial}{\partial r_{2}}-\frac{1}{r_{2}^{2}} \frac{\partial^{2}}{\partial \theta_{2}^{2}}
$$

and

$$
\begin{aligned}
-\left(\frac{\partial^{2}}{\partial r_{2}^{2}}+\frac{1}{r_{2}} \frac{\partial}{\partial r_{2}}+\right. & \left.\frac{1}{r_{2}^{2}} \frac{\partial^{2}}{\partial \theta_{2}^{2}}\right)\left(\tilde{K}_{n}\left(2 r_{2}\right)\left(\xi_{3} \pm i \xi_{4}\right)^{k}\right) \\
& =4\left((k+1) \tilde{K}_{n+1}\left(2 r_{2}\right)-\right. \\
& \left.r_{2}^{2} \tilde{K}_{n+2}\left(2 r_{2}\right)\right)\left(\xi_{3} \pm i \xi_{4}\right)^{k} \\
& =4\left((k-n) \tilde{K}_{n+1}\left(2 r_{2}\right)-\tilde{K}_{n}\left(2 r_{2}\right)\right)\left(\xi_{3} \pm i \xi_{4}\right)^{k}
\end{aligned}
$$

We can realize $r^{l+k} \tilde{K}_{n}(2 r) e^{ \pm i l \theta_{1}} e^{ \pm i k \theta_{2}}$ as the restriction of $\tilde{K}_{n}\left(2 r_{2}\right)\left(\xi_{1} \pm i \xi_{2}\right)^{l}\left(\xi_{3} \pm i \xi_{4}\right)^{k}$ to the 
cone $C^{*}$. Then

$$
\begin{aligned}
& P_{1}\left(\tilde{K}_{n}\left(2 r_{2}\right)\left(\xi_{1} \pm i \xi_{2}\right)^{l}\left(\xi_{3} \pm i \xi_{4}\right)^{k}\right) \\
& =-\left(\xi_{1}\left(\frac{\partial^{2}}{\partial r_{2}^{2}}+\frac{1}{r_{2}} \frac{\partial}{\partial r_{2}}+\frac{1}{r_{2}^{2}} \frac{\partial^{2}}{\partial \theta_{2}^{2}}\right)+2 l \widetilde{\operatorname{deg}}\right)\left(\tilde{K}_{n}\left(2 r_{2}\right)\left(\xi_{1} \pm i \xi_{2}\right)^{l-1}\left(\xi_{3} \pm i \xi_{4}\right)^{k}\right) \\
& =4 \xi_{1}\left((k-n) \tilde{K}_{n+1}\left(2 r_{2}\right)-\tilde{K}_{n}\left(2 r_{2}\right)\right)\left(\xi_{1} \pm i \xi_{2}\right)^{l}\left(\xi_{3} \pm i \xi_{4}\right)^{k} \\
& -2 l\left((l+k) \tilde{K}_{n}\left(2 r_{2}\right)-2 r_{2}^{2} \tilde{K}_{n+1}\left(2 r_{2}\right)\right)\left(\xi_{1} \pm i \xi_{2}\right)^{l-1}\left(\xi_{3} \pm i \xi_{4}\right)^{k} \\
& =2\left((k-n) \tilde{K}_{n+1}\left(2 r_{2}\right)-\tilde{K}_{n}\left(2 r_{2}\right)\right)\left(\xi_{1} \pm i \xi_{2}\right)^{l+1}\left(\xi_{3} \pm i \xi_{4}\right)^{k} \\
& +2\left(n(k-n) \tilde{K}_{n}\left(2 r_{2}\right)+(k-2 n+1) \tilde{K}_{n-1}\left(2 r_{2}\right)-\tilde{K}_{n-2}\left(2 r_{2}\right)\right)\left(\xi_{1} \pm i \xi_{2}\right)^{l-1}\left(\xi_{3} \pm i \xi_{4}\right)^{k} \\
& +2 l\left((2 n-l-k) \tilde{K}_{n}\left(2 r_{2}\right)+2 \tilde{K}_{n-1}\left(2 r_{2}\right)\right)\left(\xi_{1} \pm i \xi_{2}\right)^{l-1}\left(\xi_{3} \pm i \xi_{4}\right)^{k} \\
& =2\left((k-n) \tilde{K}_{n+1}\left(2 r_{2}\right)-\tilde{K}_{n}\left(2 r_{2}\right)\right)\left(\xi_{1} \pm i \xi_{2}\right)^{l+1}\left(\xi_{3} \pm i \xi_{4}\right)^{k} \\
& +2\left((n-l)(l+k-n) \tilde{K}_{n}\left(2 r_{2}\right)+(2 l+k-2 n+1) \tilde{K}_{n-1}\left(2 r_{2}\right)-\tilde{K}_{n-2}\left(2 r_{2}\right)\right)\left(\xi_{1} \pm i \xi_{2}\right)^{l-1}\left(\xi_{3} \pm i \xi_{4}\right)^{k} \text {; } \\
& P_{2}\left(\tilde{K}_{n}\left(2 r_{2}\right)\left(\xi_{1} \pm i \xi_{2}\right)^{l}\left(\xi_{3} \pm i \xi_{4}\right)^{k}\right) \\
& =-\left(\xi_{2}\left(\frac{\partial^{2}}{\partial r_{2}^{2}}+\frac{1}{r_{2}} \frac{\partial}{\partial r_{2}}+\frac{1}{r_{2}^{2}} \frac{\partial^{2}}{\partial \theta_{2}^{2}}\right) \pm 2 l \widetilde{\mathrm{deg}}\right)\left(\tilde{K}_{n}\left(2 r_{2}\right)\left(\xi_{1} \pm i \xi_{2}\right)^{l-1}\left(\xi_{3} \pm i \xi_{4}\right)^{k}\right) \\
& =4 \xi_{2}\left((k-n) \tilde{K}_{n+1}\left(2 r_{2}\right)-\tilde{K}_{n}\left(2 r_{2}\right)\right)\left(\xi_{1} \pm i \xi_{2}\right)^{l}\left(\xi_{3} \pm i \xi_{4}\right)^{k} \\
& \mp 2 l i\left((l+k) \tilde{K}_{n}\left(2 r_{2}\right)-2 r_{2}^{2} \tilde{K}_{n+1}\left(2 r_{2}\right)\right)\left(\xi_{1} \pm i \xi_{2}\right)^{l-1}\left(\xi_{3} \pm i \xi_{4}\right)^{k} \\
& =\mp 2 i\left((k-n) \tilde{K}_{n+1}\left(2 r_{2}\right)-\tilde{K}_{n}\left(2 r_{2}\right)\right)\left(\xi_{1} \pm i \xi_{2}\right)^{l+1}\left(\xi_{3} \pm i \xi_{4}\right)^{k} \\
& \pm 2 i\left(n(k-n) \tilde{K}_{n}\left(2 r_{2}\right)+(k-2 n+1) \tilde{K}_{n-1}\left(2 r_{2}\right)-\tilde{K}_{n-2}\left(2 r_{2}\right)\right)\left(\xi_{1} \pm i \xi_{2}\right)^{l-1}\left(\xi_{3} \pm i \xi_{4}\right)^{k} \\
& \pm 2 l i\left((2 n-l-k) \tilde{K}_{n}\left(2 r_{2}\right)+2 \tilde{K}_{n-1}\left(2 r_{2}\right)\right)\left(\xi_{1} \pm i \xi_{2}\right)^{l-1}\left(\xi_{3} \pm i \xi_{4}\right)^{k} \\
& =\mp 2 i\left((k-n) \tilde{K}_{n+1}\left(2 r_{2}\right)-\tilde{K}_{n}\left(2 r_{2}\right)\right)\left(\xi_{1} \pm i \xi_{2}\right)^{l+1}\left(\xi_{3} \pm i \xi_{4}\right)^{k} \\
& \pm 2 i\left((n-l)(l+k-n) \tilde{K}_{n}\left(2 r_{2}\right)+(2 l+k-2 n+1) \tilde{K}_{n-1}\left(2 r_{2}\right)-\tilde{K}_{n-2}\left(2 r_{2}\right)\right)\left(\xi_{1} \pm i \xi_{2}\right)^{l-1}\left(\xi_{3} \pm i \xi_{4}\right)^{k} \text {. }
\end{aligned}
$$

These calculations prove that $\mathfrak{s l}(4, \mathbb{R})$ preserves the space of finite linear combinations of the functions (6).

Proposition 3 The functions $\tilde{K}_{n}(2 r)\left(\xi_{1} \pm i \xi_{2}\right)^{l}\left(\xi_{3} \pm i \xi_{4}\right)^{k}, k, l \geq 0$ and $-\infty<n \leq \min (k, l)$, are the $K$-finite vectors for the action of $\mathfrak{s l}(4, \mathbb{R})$. Here we identify $\mathfrak{s l}(4, \mathbb{R})$ with $\mathfrak{s o}(3,3)$ and take the maximal compact subgroup corresponding to $\mathfrak{s o}(3) \times \mathfrak{s o}(3)$.

Proof. The Lie algebra $\mathfrak{s o}(3) \times \mathfrak{s o}(3)$ is generated by $\mathfrak{s o}(2) \times \mathfrak{s o}(2)$ and elements $N_{j}+\varepsilon_{j} \overline{N_{j}}$, $1 \leq j \leq 4$. Then $\mathfrak{s o}(2) \times \mathfrak{s o}(2)$ acts by linear combinations of $\partial / \partial \theta_{1}$ and $\partial / \partial \theta_{2}$, and $N_{j}+\varepsilon_{j} \overline{N_{j}}$ acts by $2 i \xi_{j}+\frac{i}{2} P_{j}, 1 \leq j \leq 4$.

$$
\begin{aligned}
\left(2\left(\xi_{1}+i \xi_{2}\right)+\right. & \left.\frac{1}{2}\left(P_{1}+i P_{2}\right)\right)\left(\tilde{K}_{n}(2 r)\left(\xi_{1}+i \xi_{2}\right)^{l}\left(\xi_{3} \pm i \xi_{4}\right)^{k}\right) \\
& =2(k-n) \tilde{K}_{n+1}(2 r)\left(\xi_{1}+i \xi_{2}\right)^{l+1}\left(\xi_{3} \pm i \xi_{4}\right)^{k}
\end{aligned}
$$




$$
\begin{aligned}
\left(2\left(\xi_{1}-i \xi_{2}\right)+\right. & \left.\frac{1}{2}\left(P_{1}-i P_{2}\right)\right)\left(\tilde{K}_{n}(2 r)\left(\xi_{1}+i \xi_{2}\right)^{l}\left(\xi_{3} \pm i \xi_{4}\right)^{k}\right) \\
= & 2\left((n-l)(l+k-n) \tilde{K}_{n}(2 r)+(2 l+k-n) \tilde{K}_{n-1}(2 r)\right)\left(\xi_{1}+i \xi_{2}\right)^{l-1}\left(\xi_{3} \pm i \xi_{4}\right)^{k} \\
\left(2\left(\xi_{1}-i \xi_{2}\right)+\right. & \left.\frac{1}{2}\left(P_{1}-i P_{2}\right)\right)\left(\tilde{K}_{n}(2 r)\left(\xi_{1}-i \xi_{2}\right)^{l}\left(\xi_{3} \pm i \xi_{4}\right)^{k}\right) \\
& =2(k-n) \tilde{K}_{n+1}(2 r)\left(\xi_{1}-i \xi_{2}\right)^{l+1}\left(\xi_{3} \pm i \xi_{4}\right)^{k} .
\end{aligned}
$$

Thus $\mathfrak{s o}(3) \times \mathfrak{s o}(3)$ acts on a function of type (6) finitely if $n \leq \min (k, l)$. Lemma 3.4.1 in [KM] implies that these functions belong to $L^{2}\left(C^{*}\right)$.

We get all the $K$-finite vectors this way because setting $n=\min (k, l)$ we obtain the highest weight vector from each $K$-type of $L^{2}\left(C^{*}\right)$ by Theorem 3.1.1 in [KM].

\section{$5 \quad$ Fourier Transforms of $\frac{1}{\left(N(X) \pm R^{2} \pm i 0\right)^{2}}$}

As a preparation for the proof of Theorem 2, in this section we compute the Fourier transforms of the distributions $\left(N(X) \pm R^{2} \pm i 0\right)^{-2}$ on $\mathbb{H}_{\mathbb{R}}$. The following proposition is closely related to the formulas from $\S 2.8$, Chapter III of [GS], which describe the Fourier transforms of the generalized functions $\left(R^{2}+Q \pm i 0\right)^{\lambda}$ associated to a quadratic form $Q$ on $\mathbb{R}^{n}$, where $\operatorname{Re} \lambda<-n / 2$. However, in our case $n=4$ and $\lambda=-2=-n / 2$, and we can think of it as a "limit" of the setting of [GS]. Recall that the Fourier transform is normalized as in (2), and let $J_{0}$ denote the Bessel function of the first kind.

Proposition 4 Let $R>0$, then

$$
\begin{gathered}
\lim _{\varepsilon \rightarrow 0^{+}} \frac{1}{4 \pi^{2}} \int_{X \in \mathbb{H}_{\mathbb{R}}} \frac{e^{i \xi \cdot X} d V}{\left(N(X)-R^{2} \pm i \varepsilon\right)^{2}}= \begin{cases}\frac{\pi}{4} Y_{0}(R \sqrt{\langle\xi, \xi\rangle}) \pm i \frac{\pi}{4} J_{0}(R \sqrt{\langle\xi, \xi\rangle}) & \langle\xi, \xi\rangle>0 ; \\
-\frac{1}{2} K_{0}(R \sqrt{-\langle\xi, \xi\rangle}) & \langle\xi, \xi\rangle<0 ;\end{cases} \\
\lim _{\varepsilon \rightarrow 0^{+}} \frac{1}{4 \pi^{2}} \int_{X \in \mathbb{H}_{\mathbb{R}}} \frac{e^{i \xi \cdot X} d V}{\left(N(X)+R^{2} \pm i \varepsilon\right)^{2}}= \begin{cases}-\frac{1}{2} K_{0}(R \sqrt{\langle\xi, \xi\rangle}) & \langle\xi, \xi\rangle>0 ; \\
\frac{\pi}{4} Y_{0}(R \sqrt{-\langle\xi, \xi\rangle}) \mp i \frac{\pi}{4} J_{0}(R \sqrt{-\langle\xi, \xi\rangle}) & \langle\xi, \xi\rangle<0 .\end{cases}
\end{gathered}
$$

Proof. We use the following integral representations of the Bessel functions that can be found, for instance, in $[\mathrm{Er}$ or $\mathrm{GR}$ :

$$
\begin{gathered}
J_{0}(u)=\frac{2}{\pi} \int_{0}^{\infty} \sin (u \cosh t) d t, \quad Y_{0}(u)=-\frac{2}{\pi} \int_{0}^{\infty} \cos (u \cosh t) d t \\
K_{0}(u)=\int_{0}^{\infty} \cos (u \sinh t) d t=\int_{0}^{\infty} \exp (-u \cosh t) d t .
\end{gathered}
$$

Write $\xi=\left(\xi_{1}, \xi_{2}, \xi_{3}, \xi_{4}\right)$ and let $r_{1}=\sqrt{\left(\xi_{1}\right)^{2}+\left(\xi_{2}\right)^{2}}, r_{2}=\sqrt{\left(\xi_{3}\right)^{2}+\left(\xi_{4}\right)^{2}}$. Rotating $\xi$ if necessary, without loss of generality we can assume that $\xi=\left(r_{1}, 0, r_{2}, 0\right)$. Using the formulas

$$
\int \frac{d x}{\left(a^{2}-x^{2}\right)^{2}}=\frac{x}{2 a^{2}\left(a^{2}-x^{2}\right)}+\frac{1}{4 a^{3}} \log \left(\frac{x+a}{x-a}\right)+C, \quad \int \frac{d x}{\left(a^{2}+x^{2}\right)^{3 / 2}}=\frac{x}{a^{2} \sqrt{a^{2}+x^{2}}}+C,
$$

we compute:

$$
\begin{aligned}
\lim _{\varepsilon \rightarrow 0^{+}} \iiint \int_{\mathbb{R}^{4}} \frac{e^{i\left(r_{1} x_{1}+r_{2} x_{3}\right)}}{\left(x_{1}^{2}+x_{2}^{2}-x_{3}^{2}-x_{4}^{2}-R^{2}+i \varepsilon\right)^{2}} d x_{1} d x_{2} d x_{3} d x_{4} \\
=-\frac{\pi i}{2} \lim _{\varepsilon \rightarrow 0^{+}} \iiint_{\mathbb{R}^{3}} \frac{e^{i\left(r_{1} x_{1}+r_{2} x_{3}\right)}}{\left(x_{1}^{2}+x_{2}^{2}-x_{3}^{2}-R^{2}+i \varepsilon\right)^{3 / 2}} d x_{1} d x_{2} d x_{3} \\
=-\pi i \lim _{\varepsilon \rightarrow 0^{+}} \iint_{\mathbb{R}^{2}} \frac{e^{i\left(r_{1} x_{1}+r_{2} x_{3}\right)}}{x_{1}^{2}-x_{3}^{2}-R^{2}+i \varepsilon} d x_{1} d x_{3} .
\end{aligned}
$$


Then we divide $\mathbb{R}^{2}$ into two regions: $\left\{\left|x_{1}\right|>\left|x_{3}\right|\right\}$ and $\left\{\left|x_{1}\right|<\left|x_{3}\right|\right\}$. On the first region we change the coordinates $\left(x_{1}, x_{3}\right)$ to $(s, t)$ so that

$$
x_{1}=R s \cosh t, \quad x_{3}=R s \sinh t, \quad-\infty<s<\infty,-\infty<t<\infty,
$$

and on the second region we change the coordinates $\left(x_{1}, x_{3}\right)$ to $(s, t)$ so that

$$
x_{1}=R s \sinh t, \quad x_{3}=R s \cosh t, \quad-\infty<s<\infty,-\infty<t<\infty,
$$

and (9) reduces to

$$
\pi i \lim _{\varepsilon \rightarrow 0^{+}} \iint_{\mathbb{R}^{2}}|s|\left(\frac{\exp \left(i R s\left(r_{1} \cosh t+r_{2} \sinh t\right)\right)}{1-s^{2}-i \varepsilon}+\frac{\exp \left(i R s\left(r_{1} \sinh t+r_{2} \cosh t\right)\right)}{1+s^{2}-i \varepsilon}\right) d s d t .
$$

If $r_{1}>r_{2}$, then, for some $\tau \geq 0$, we can write

$$
r_{1}=\sqrt{r_{1}^{2}-r_{2}^{2}} \cdot \cosh \tau, \quad r_{2}=\sqrt{r_{1}^{2}-r_{2}^{2}} \cdot \sinh \tau,
$$

and

$$
\begin{gathered}
\int_{\mathbb{R}} \exp \left(i R s\left(r_{1} \sinh t+r_{2} \cosh t\right)\right) d t=\int_{\mathbb{R}} \exp \left(i R s \sqrt{r_{1}^{2}-r_{2}^{2}} \sinh (t+\tau)\right) d t \\
=\int_{\mathbb{R}} \cos \left(R s \sqrt{r_{1}^{2}-r_{2}^{2}} \sinh (t+\tau)\right) d t+i \int_{\mathbb{R}} \sin \left(R s \sqrt{r_{1}^{2}-r_{2}^{2}} \sinh (t+\tau)\right) d t \\
=2 K_{0}\left(R s \sqrt{r_{1}^{2}-r_{2}^{2}}\right)=\int_{\mathbb{R}} \exp \left(-R|s| \sqrt{r_{1}^{2}-r_{2}^{2}} \cosh (t+\tau)\right) d t \\
=\int_{\mathbb{R}} \exp \left(-R|s|\left(r_{1} \cosh t+r_{2} \sinh t\right)\right) d t
\end{gathered}
$$

Thus we can rewrite (10) as

$$
\begin{aligned}
& \pi i \lim _{\varepsilon \rightarrow 0^{+}} \int_{\mathbb{R}}\left(\int_{\Gamma_{1}} \frac{s \exp \left(i R s\left(r_{1} \cosh t+r_{2} \sinh t\right)\right)}{1-s^{2}-i \varepsilon} d s\right. \\
&\left.+\int_{\Gamma_{2}} \frac{s \exp \left(-i R s\left(r_{1} \cosh t+r_{2} \sinh t\right)\right)}{1-s^{2}-i \varepsilon} d s\right) d t
\end{aligned}
$$

where $\Gamma_{1}$ is the contour in the complex plane $\mathbb{C}$ which starts at infinity, goes along the positive imaginary axis towards the origin and then along the positive real axis towards infinity, and $\Gamma_{2}$ is the contour which starts at infinity, goes along the negative imaginary axis towards the origin and then along the positive real axis towards infinity. We can compute the integrals with respect to $s$ using residues:

$$
\begin{aligned}
& \lim _{\varepsilon \rightarrow 0^{+}} \int_{\mathbb{H}_{\mathbb{R}}} \frac{e^{i\left(r_{1} x_{1}+r_{2} x_{3}\right)}}{\left(N(X)-R^{2}+i \varepsilon\right)^{2}} d V=-\pi^{2} \int_{\mathbb{R}} \exp \left(-i R\left(r_{1} \cosh t+r_{2} \sinh t\right)\right) d t \\
&=-\pi^{2} \int_{\mathbb{R}} \cos \left(R \sqrt{r_{1}^{2}-r_{2}^{2}} \cosh (t+\tau)\right) d t+i \pi^{2} \int_{\mathbb{R}} \sin \left(R \sqrt{r_{1}^{2}-r_{2}^{2}} \cosh (t+\tau)\right) d t \\
&=\pi^{3} Y_{0}\left(R \sqrt{r_{1}^{2}-r_{2}^{2}}\right)+i \pi^{3} J_{0}\left(R \sqrt{r_{1}^{2}-r_{2}^{2}}\right) .
\end{aligned}
$$

If $r_{1}<r_{2}$, then, for some $\tau \geq 0$, we can write

$$
r_{1}=\sqrt{r_{2}^{2}-r_{1}^{2}} \cdot \sinh \tau, \quad r_{2}=\sqrt{r_{2}^{2}-r_{1}^{2}} \cdot \cosh \tau
$$


and

$$
\begin{gathered}
\int_{\mathbb{R}} \exp \left(i R s\left(r_{1} \cosh t+r_{2} \sinh t\right)\right) d t=\int_{\mathbb{R}} \exp \left(i R s \sqrt{r_{2}^{2}-r_{1}^{2}} \sinh (t+\tau)\right) d t \\
=\int_{\mathbb{R}} \cos \left(R s \sqrt{r_{2}^{2}-r_{1}^{2}} \sinh (t+\tau)\right) d t+i \int_{\mathbb{R}} \sin \left(R s \sqrt{r_{2}^{2}-r_{1}^{2}} \sinh (t+\tau)\right) d t \\
=2 K_{0}\left(R s \sqrt{r_{2}^{2}-r_{1}^{2}}\right)=\int_{\mathbb{R}} \exp \left(-R|s| \sqrt{r_{2}^{2}-r_{1}^{2}} \cosh (t+\tau)\right) d t \\
=\int_{\mathbb{R}} \exp \left(-R|s|\left(r_{1} \sinh t+r_{2} \cosh t\right)\right) d t
\end{gathered}
$$

Thus we can rewrite (10) as

$$
\begin{aligned}
& \pi i \lim _{\varepsilon \rightarrow 0^{+}} \int_{\mathbb{R}}\left(\int_{\Gamma_{1}} \frac{s \exp \left(i R s\left(r_{1} \sinh t+r_{2} \cosh t\right)\right)}{1+s^{2}-i \varepsilon} d s\right. \\
&\left.+\int_{\Gamma_{2}} \frac{s \exp \left(-i R s\left(r_{1} \sinh t+r_{2} \cosh t\right)\right)}{1+s^{2}-i \varepsilon} d s\right) d t
\end{aligned}
$$

where $\Gamma_{1}$ and $\Gamma_{2}$ are the same contours in the complex plane $\mathbb{C}$ as above. Using residues to compute the integrals with respect to $s$ we get:

$$
\begin{gathered}
\lim _{\varepsilon \rightarrow 0^{+}} \int_{\mathbb{H}_{\mathbb{R}}} \frac{e^{i\left(r_{1} x_{1}+r_{2} x_{3}\right)}}{\left(N(X)-R^{2}+i \varepsilon\right)^{2}} d V=-\pi^{2} \int_{\mathbb{R}} \exp \left(-R\left(r_{1} \sinh t+r_{2} \cosh t\right)\right) d t \\
\left.=-\pi^{2} \int_{\mathbb{R}} \exp \left(-R \sqrt{r_{2}^{2}-r_{1}^{2}} \cosh (t+\tau)\right)\right) d t=-2 \pi^{2} K_{0}\left(R \sqrt{r_{2}^{2}-r_{1}^{2}}\right) .
\end{gathered}
$$

The other Fourier transforms are found in exactly the same way.

Corollary 5 Let $R>0$ and $\xi, \xi^{\prime} \in C^{*}$, then

$$
\begin{gathered}
\lim _{\varepsilon \rightarrow 0^{+}} \frac{1}{4 \pi^{2}} \int_{X \in \mathbb{H}_{\mathbb{R}}}\left(\frac{e^{i\left(\xi-\xi^{\prime}\right) \cdot X}}{(N(X)-4+i \varepsilon)^{2}}+\frac{e^{i\left(\xi-\xi^{\prime}\right) \cdot X}}{(N(X)-4-i \varepsilon)^{2}}\right) d V=\frac{\pi}{2} \cdot \Psi_{0}\left(-\left\langle\xi, \xi^{\prime}\right\rangle\right), \\
\lim _{\varepsilon \rightarrow 0^{+}} \frac{1}{4 \pi^{2}} \int_{X \in \mathbb{H}_{\mathbb{R}}}\left(\frac{e^{i\left(\xi-\xi^{\prime}\right) \cdot X}}{\left(N(X)-R^{2}+i \varepsilon\right)^{2}}-\frac{e^{i\left(\xi-\xi^{\prime}\right) \cdot X}}{\left(N(X)-R^{2}-i \varepsilon\right)^{2}}\right) d V \\
=\left\{\begin{array}{l}
0 \\
\frac{\pi i}{2} J_{0}\left(R \sqrt{-2\left\langle\xi, \xi^{\prime}\right\rangle}\right) \\
\left\langle\xi, \xi^{\prime}\right\rangle<0
\end{array}=\frac{\pi i}{2} \cdot \Phi_{0}^{+}\left(-\frac{R^{2}}{4}\left\langle\xi, \xi^{\prime}\right\rangle\right) .\right.
\end{gathered}
$$

Proof. Since $\xi, \xi^{\prime} \in C^{*},\left\langle\xi-\xi^{\prime}, \xi-\xi^{\prime}\right\rangle=-2\left\langle\xi, \xi^{\prime}\right\rangle$, and the result follows from the above proposition.

We conclude this section with the following expressions for the kernels $\Psi_{0}\left(\left\langle\xi, \xi^{\prime}\right\rangle\right)$ and $\Phi_{0}^{+}\left(\left\langle\xi, \xi^{\prime}\right\rangle\right)$ defined by (3) and (11).

Lemma 6 Let $R>0, \xi=\left(\xi_{1}, \xi_{2}, \xi_{3}, \xi_{3}\right), \xi^{\prime}=\left(\xi_{1}^{\prime}, \xi_{2}^{\prime}, \xi_{3}^{\prime}, \xi_{3}^{\prime}\right) \in C^{*}$,

$$
r_{1}=\sqrt{\left(\xi_{1}-\xi_{1}^{\prime}\right)^{2}+\left(\xi_{2}-\xi_{2}^{\prime}\right)^{2}}, \quad r_{2}=\sqrt{\left(\xi_{3}-\xi_{3}^{\prime}\right)^{2}+\left(\xi_{4}-\xi_{4}^{\prime}\right)^{2}},
$$


then

$$
\begin{aligned}
\Psi_{0}\left(\frac{R^{2}}{4}\left\langle\xi, \xi^{\prime}\right\rangle\right) & =-\frac{1}{\pi} \int_{-\infty}^{\infty} \cos \left(R r_{1} \sinh t+R r_{2} \cosh t\right) d t \\
\Psi_{0}\left(-\frac{R^{2}}{4}\left\langle\xi, \xi^{\prime}\right\rangle\right) & =-\frac{1}{\pi} \int_{-\infty}^{\infty} \cos \left(R r_{1} \cosh t+R r_{2} \sinh t\right) d t \\
\Phi_{0}^{+}\left(\frac{R^{2}}{4}\left\langle\xi, \xi^{\prime}\right\rangle\right) & =\frac{1}{\pi} \int_{-\infty}^{\infty} \sin \left(R r_{1} \sinh t+R r_{2} \cosh t\right) d t \\
\Phi_{0}^{+}\left(-\frac{R^{2}}{4}\left\langle\xi, \xi^{\prime}\right\rangle\right) & =\frac{1}{\pi} \int_{-\infty}^{\infty} \sin \left(R r_{1} \cosh t+R r_{2} \sinh t\right) d t .
\end{aligned}
$$

Since we are not going to use this lemma, we omit its proof.

\section{Fourier Transform of $\mathrm{Pl}_{R}^{\prime}$ and the Proof of Theorem 2}

In this section we compute the Fourier transform of $\mathrm{Pl}_{R}^{\prime}$, compare it with $\mathcal{F}_{C^{*}}^{\prime}$ and use that comparison to finish the proof of Theorem 2. As in [KM], let $T: L^{2}\left(C^{*}\right) \rightarrow \mathcal{S}^{\prime}\left(\mathbb{H}_{\mathbb{R}}^{*}\right)$ be the continuous injective map sending a function $f \in L^{2}\left(C^{*}\right)$ into a distribution $f \cdot \delta\left(C^{*}\right)$ belonging to the space of tempered distributions on $\mathbb{H}_{\mathbb{R}}^{*}$. If a function $\varphi$ on $\mathbb{H}_{\mathbb{R}}$ satisfies $\square_{2,2} \varphi=0$, its Fourier transform $\hat{\varphi}$ is a distribution supported on the cone $C^{*}$. As explained in [KM], [KØ], if this function $\varphi$ lies in the solution model for minimal representation $V^{3,3}$ of $O(3,3)$, then $\hat{\varphi}$ lies in the image $T\left(L^{2}\left(C^{*}\right)\right)$, and so it makes sense to talk about $T^{-1}(\hat{\varphi}) \in L^{2}\left(C^{*}\right)$. Let $F T: V^{3,3} \rightarrow L^{2}\left(C^{*}\right)$ denote the map $\varphi \mapsto T^{-1}(\hat{\varphi})$. Then we have a commutative diagram

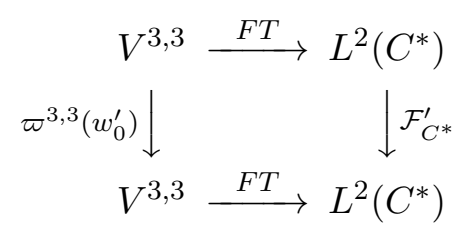

As a representation of $S U(1,1) \times S U(1,1)$, which is a double cover of $S O(2,2), L^{2}\left(C^{*}\right)$ is a direct integral of the spaces of homogeneous functions of degree $-1+i \rho$ with $\rho \in \mathbb{R}$. Then $F T^{-1}\left(L^{2}\left(C^{*}\right)\right)$ is also a direct integral of the spaces of homogeneous functions of degree $-1+i \rho$, $\rho \in \mathbb{R}$, satisfying $\square_{2,2} \varphi=0$. By [St] these functions restrict to the continuous spectrum functions on $S U(1,1)$ realized as the unit hyperboloid in $\mathbb{H}_{\mathbb{R}}$. This proves that the minimal representation $V^{3,3}$ is nothing but the closure of the space generated by the wave packets of matrix coefficients $t_{n \underline{m}}^{l}$ 's of $S U(1,1)$ with $\operatorname{Re} l=-1 / 2$.

Since the Fourier transform replaces products with convolutions and vice versa, we obtain the following integral expression for the operator $\varpi^{3,3}\left(w_{0}^{\prime}\right)$ in the solution model:

$$
\begin{aligned}
\varphi(X) & \mapsto\left(\varpi^{3,3}\left(w_{0}^{\prime}\right) \varphi\right)(W) \\
& =-\frac{2}{\pi^{2}} \lim _{\varepsilon \rightarrow 0^{+}} \int_{X \in C}\left(\frac{1}{(N(W-X)-4+i \varepsilon)^{2}}+\frac{1}{(N(W-X)-4-i \varepsilon)^{2}}\right) \varphi(W-X) \frac{d S}{\|X\|} .
\end{aligned}
$$

Here we used that the Fourier transform of $\delta(C)$ is $\delta\left(C^{*}\right)$, where

$$
\delta\left(C^{*}\right): \quad \psi \mapsto \frac{1}{2} \int_{\xi \in C^{*}} \psi(\xi) \frac{d S}{\|\xi\|}
$$

(see [GS], Chapter III, Section 2.6). 
Next we define a map $\widehat{\mathrm{Pl}}_{R}^{\prime}$ on $L^{2}\left(C^{*}\right)$ by

$$
\left(\widehat{\mathrm{Pl}}_{R}^{\prime} f\right)(\xi)=\frac{i}{4 \pi} \int_{\xi^{\prime} \in C^{*}} \Phi_{0}^{+}\left(-\frac{R^{2}}{4}\left\langle\xi, \xi^{\prime}\right\rangle\right) \cdot f\left(\xi^{\prime}\right) \frac{d S}{\left\|\xi^{\prime}\right\|}, \quad f \in L^{2}\left(C^{*}\right),
$$

where

$$
\Phi_{0}^{+}(t)= \begin{cases}J_{0}(2 \sqrt{2 t}) & t>0 \\ 0 & t<0\end{cases}
$$

Lemma 7 The map $\widehat{\mathrm{Pl}}_{R}^{\prime}$ sends $L^{2}\left(C^{*}\right)$ into $L^{2}\left(C^{*}\right)$.

Proof. Recall the Hilbert space isomorphism (5), hence

$$
L^{2}\left(C^{*}\right) \simeq \widehat{\bigoplus}_{l, k \in \mathbb{Z}} L^{2}\left(\mathbb{R}_{+}, \frac{r}{2} d r\right) \otimes \mathbb{C} e^{i \theta_{1}} \otimes \mathbb{C} e^{i \theta_{2}} .
$$

Note that the map $\widehat{\mathrm{Pl}}_{R}^{\prime}$ is $S^{1} \times S^{1}$-equivariant. Let $\left(\widehat{\mathrm{Pl}}_{R}^{\prime}\right)_{k, l}$ denote the restriction of $\widehat{\mathrm{Pl}}_{R}^{\prime}$ to $L^{2}\left(\mathbb{R}_{+}, \frac{r}{2} d r\right) \otimes \mathbb{C} e^{i \theta_{1}} \otimes \mathbb{C} e^{i \theta_{2}}$. Thus, $\left(\widehat{\mathrm{Pl}}_{R}^{\prime}\right)_{k, l}$ is essentially a map from functions on $\mathbb{R}_{+}$ into functions on $\mathbb{R}_{+}$, and, clearly, $\left(\widehat{\mathrm{Pl}}_{R}^{\prime}\right)_{k, l}$ is well-defined on smooth compactly supported functions on $\mathbb{R}_{+}$. The fact that $\left(\widehat{\mathrm{Pl}}_{R}^{\prime}\right)_{k, l}$ extends to $L^{2}\left(\mathbb{R}_{+}, \frac{r}{2} d r\right)$ and that its image is contained in $L^{2}\left(\mathbb{R}_{+}, \frac{r}{2} d r\right)$ is proven in Section 5.4 of $\left[\mathrm{KM}\right.$ because $\Phi_{0}^{+}(t)$ is the integral kernel of the operator $\mathcal{F}_{C^{*}}$ for the Minkowski space $\mathbb{M}$ (i.e. the case $p=4, q=2$ ).

The same reasoning as above shows that the map $\mathrm{Pl}_{R}^{\prime}$ restricted to $V^{3,3}$ can be fit into a diagram

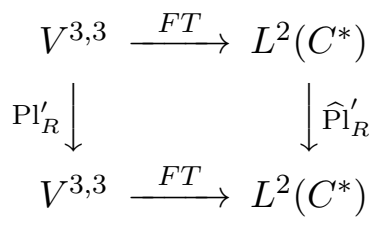

Note that if $\varphi$ is a function on $\mathbb{H}_{\mathbb{R}}$ which is homogeneous of degree $-1+i \rho$, then $F T \varphi$ is homogeneous of degree $-1-i \rho$, and both $\widehat{\mathrm{Pl}}_{R}^{\prime}(F T \varphi), \mathcal{F}_{C^{*}}^{\prime}(F T \varphi)$ are homogeneous of degree $-1+i \rho$. The most obvious thing to do is to apply operators $\widehat{\mathrm{Pl}}_{R}^{\prime}$ and $\mathcal{F}_{C^{*}}^{\prime}$ to functions $f$ on $C^{*}$ that are homogeneous of degree $1-i \rho, \rho \in \mathbb{R}$, then find the ratio $\left(\widehat{\mathrm{Pl}}_{R}^{\prime} f\right) /\left(\mathcal{F}_{C^{*}}^{\prime} f\right)$ and compare it with (11). Unfortunately, such functions are not in $L^{2}\left(C^{*}\right)$ and we would run into convergence problems.

Fix any $\xi \in C^{*} \backslash\{0\}$ and $\varepsilon \in\{0,1\}$. We identify $C^{*}$ with $\mathbb{R}_{+} \times S^{1} \times S^{1}$. Let $\psi$ be a smooth function on $S^{1} \times S^{1}$ such that $\psi\left(-\theta_{1},-\theta_{2}\right)=(-1)^{\varepsilon} \psi\left(\theta_{1}, \theta_{2}\right)$. We shall regard $\psi$ as a function on $C^{*}$ and assume that $\psi$ satisfies

$$
\operatorname{supp} \psi \cap\left\{\xi^{\prime} \in C^{*} ;\left\langle\xi, \xi^{\prime}\right\rangle=1\right\}
$$

is compact. We define a function $f_{\xi, \varepsilon}$ on $C^{*}$ by

$$
f_{\xi, \varepsilon}\left(\xi^{\prime}\right)=\psi \cdot\left|\left\langle\xi, \xi^{\prime}\right\rangle\right|^{-1 / 2} \cdot \exp \left(-\left|\left\langle\xi, \xi^{\prime}\right\rangle\right|\right)
$$

By design, $f_{\xi, \varepsilon}$ belongs to $L^{2}\left(C^{*}\right)$. Next we compute $\widehat{\mathrm{Pl}}_{R}^{\prime} f_{\xi, \varepsilon}(s \xi)$ and $\mathcal{F}_{C^{*}}^{\prime} f_{\xi, \varepsilon}(s \xi), s \in \mathbb{R}$. For this purpose we use the following integrals, which can be found in [GR] (special cases of $2.667(7-8))$ :

$$
\int_{0}^{\infty} t^{2} e^{-a t} \sin (b t) d t=\frac{2 b\left(3 a^{2}-b^{2}\right)}{\left(a^{2}+b^{2}\right)^{3}}, \quad \int_{0}^{\infty} t^{2} e^{-a t} \cos (b t) d t=\frac{2 a\left(a^{2}-3 b^{2}\right)}{\left(a^{2}+b^{2}\right)^{3}}, \quad a>0
$$


and $\quad \int_{0}^{\infty} s^{\mu-1} e^{-a s} d s=a^{-\mu} \Gamma(\mu)$.

Using (17) we find that $\widehat{\mathrm{Pl}}_{R}^{\prime} f_{\xi, \varepsilon}(s \xi)$ is proportional to the integral with respect to $\theta$ (from $\theta=0$ to $\infty$ ) of

$$
\begin{aligned}
(-1)^{\varepsilon} \frac{i}{2 \pi^{2}} \int_{0}^{\infty} \sqrt{t} e^{-\sqrt{t}} \sin (R \sqrt{2 s t} \cosh \theta) d t= & (-1)^{\varepsilon} \frac{i}{\pi^{2}} \int_{0}^{\infty} t^{2} e^{-t} \sin (R \sqrt{2 s} \cosh \theta t) d t \\
& =(-1)^{\varepsilon} \frac{2 \sqrt{2} i}{\pi^{2}} \frac{3 R \cosh \theta s^{1 / 2}-2 R^{3} \cosh ^{3} \theta s^{3 / 2}}{\left(1+2 R^{2} \cosh ^{2} \theta s\right)^{3}}
\end{aligned}
$$

Similarly, using (77) and (8) we find that $\mathcal{F}_{C^{*}}^{\prime} f_{\xi, \varepsilon}(s \xi)$ is proportional to the integral with respect to $\theta$ (from $\theta=0$ to $\infty$ ) of

$$
\begin{aligned}
& \frac{2}{\pi^{2}} \int_{0}^{\infty} \sqrt{t} e^{-\sqrt{t}}(\exp (-2 \sqrt{2 s t} \cosh \theta)\left.+(-1)^{\varepsilon} \cos (2 \sqrt{2 s t} \cosh \theta)\right) d t \\
&=\frac{4}{\pi^{2}} \int_{0}^{\infty} t^{2} e^{-t}\left(\exp (-2 \sqrt{2 s} \cosh \theta t)+(-1)^{\varepsilon} \cos (2 \sqrt{2 s} \cosh \theta t)\right) d t \\
&=\frac{8}{\pi^{2}}\left(\frac{1}{(1+2 \sqrt{2 s} \cosh \theta)^{3}}+(-1)^{\varepsilon} \frac{1-24 \cosh ^{2} \theta s}{\left(1+8 \cosh ^{2} \theta s\right)^{3}}\right)
\end{aligned}
$$

Next we apply the Mellin transform $f(s) \mapsto(\mathcal{M} f)(\rho)=\int_{0}^{\infty} f(s) s^{1-i \rho} \frac{d s}{s}$. For this purpose we use integral formula 8.384 from [GR]

$$
\int_{0}^{\infty} \frac{s^{\mu-1}}{(1+a s)^{\nu}} d s=a^{-\mu} \frac{\Gamma(\mu) \Gamma(\nu-\mu)}{\Gamma(\nu)} .
$$

Thus $\mathcal{M}\left(\widehat{\mathrm{Pl}}_{R}^{\prime} f_{\xi, \varepsilon}(s \xi)\right)$ is proportional to the integral with respect to $\theta$ of

$$
\begin{aligned}
& (-1)^{\varepsilon} \frac{2 \sqrt{2} i}{\pi^{2}} \int_{0}^{\infty} \frac{3 R \cosh \theta s^{1 / 2}-2 R^{3} \cosh ^{3} \theta s^{3 / 2}}{\left(1+2 R^{2} \cosh ^{2} \theta s\right)^{3}} s^{-i \rho} d s \\
& =(-1)^{\varepsilon} \frac{i}{\pi^{2}}(\sqrt{2} R \cosh \theta)^{-2+2 i \rho}(3 \Gamma(3 / 2-i \rho) \Gamma(3 / 2+i \rho)-\Gamma(5 / 2-i \rho) \Gamma(1 / 2+i \rho)) \\
& =(-1)^{\varepsilon} \frac{i}{\pi^{2}}(\sqrt{2} R \cosh \theta)^{-2+2 i \rho} \Gamma(1 / 2-i \rho) \Gamma(1 / 2+i \rho)(1 / 2-i \rho)(3(1 / 2+i \rho)-(3 / 2-i \rho)) \\
& =(-1)^{\varepsilon+1} \frac{2}{\pi} \frac{\rho(1-2 i \rho)}{\cos (\pi i \rho)}(\sqrt{2} R \cosh \theta)^{-2+2 i \rho} .
\end{aligned}
$$

On the other hand, $\mathcal{M}\left(\mathcal{F}_{C^{*}}^{\prime} f_{\xi, \varepsilon}(s \xi)\right)$ is proportional to the integral with respect to $\theta$ of

$$
\begin{aligned}
& \frac{8}{\pi^{2}} \int_{0}^{\infty}\left(\frac{1}{(1+2 \sqrt{2 s} \cosh \theta)^{3}}+(-1)^{\varepsilon} \frac{1-24 \cosh ^{2} \theta s}{\left(1+8 \cosh ^{2} \theta s\right)^{3}}\right) s^{-i \rho} d s \\
= & \frac{4}{\pi^{2}}(2 \sqrt{2} \cosh \theta)^{-2+2 i \rho}\left(2 \Gamma(2-2 i \rho) \Gamma(1+2 i \rho)+(-1)^{\varepsilon}(\Gamma(1-i \rho) \Gamma(2+i \rho)-3 \Gamma(2-i \rho) \Gamma(1+i \rho))\right) \\
= & \frac{4 i \rho}{\pi^{2}}(2 \sqrt{2} \cosh \theta)^{-2+2 i \rho}\left(4(1-2 i \rho) \Gamma(1-2 i \rho) \Gamma(2 i \rho)+(-1)^{\varepsilon} \Gamma(1-i \rho) \Gamma(i \rho)((1+i \rho)-3(1-i \rho))\right) \\
= & \frac{8 i \rho(1-2 i \rho)}{\pi}(2 \sqrt{2} \cosh \theta)^{-2+2 i \rho}\left(\frac{2}{\sin (2 \pi i \rho)}-\frac{(-1)^{\varepsilon}}{\sin (\pi i \rho)}\right) \\
= & \frac{8 i}{\pi} \frac{\rho(1-2 i \rho)}{\cos (\pi i \rho)}(2 \sqrt{2} \cosh \theta)^{-2+2 i \rho} \cdot \begin{cases}\tan (\pi i \rho / 2) & \text { if } \varepsilon=0 ; \\
\cot (\pi i \rho / 2) & \text { if } \varepsilon=1 .\end{cases}
\end{aligned}
$$


Therefore,

$$
\begin{aligned}
\frac{\mathcal{M}\left(\widehat{\mathrm{Pl}}_{R}^{\prime} f_{\xi, \varepsilon}(s \xi)\right)}{\mathcal{M}\left(\mathcal{F}_{C^{*}}^{\prime} f_{\xi, \varepsilon}(s \xi)\right)}(\rho)=i R^{-2+2 i \rho} 2^{-2 i \rho} \cdot \begin{cases}\cot (\pi i \rho / 2) & \text { if } \varepsilon=0 \\
-\tan (\pi i \rho / 2) & \text { if } \varepsilon=1\end{cases} \\
=R^{-2+2 i \rho} 2^{-2 i \rho} \cdot \begin{cases}\operatorname{coth}(\pi \rho / 2) & \text { if } \varepsilon=0 \\
\tanh (\pi \rho / 2) & \text { if } \varepsilon=1 .\end{cases}
\end{aligned}
$$

This completes the proof of Theorem 2 ,

\section{References}

[Er] A. Erdélyi et al, Higher Transcendental Functions. Based, in part, on notes left by Harry Bateman, Vols. I, II, III, McGraw-Hill, New York-Toronto-London, 1953.

[FL1] I. Frenkel, M. Libine, Quaternionic analysis, representation theory and physics, Advances in Math 218 (2008), 1806-1877; also arXiv:0711.2699.

[FL2] I. Frenkel, M. Libine, Split quaternionic analysis and the separation of the series for $S L(2, \mathbb{R})$ and $S L(2, \mathbb{C}) / S L(2, \mathbb{R})$, Advances in Math 228 (2011), 678-763; also arXiv:1009.2532.

[GS] I. M. Gelfand, G. E. Shilov, Generalized functions. Vol. 1: Properties and Operations, translated from the Russian by E. Saletan, Academic Press, New York and London 1964.

[GR] I. S. Gradshteyn, I. M. Ryzhik, Table of Integrals, Series, and Products, 7th edition, Academic Press, Amsterdam, 2007.

[HKMM] J. Hilgert, T. Kobayashi, G. Mano and J. Möllers, Special functions associated to a certain fourth order differential equation, to appear in Ramanujan J. Math; also arXiv:0907.2608.

[KM] T. Kobayashi, G. Mano, The Schrödinger model for the minimal representation of the indefinite orthogonal group $O(p, q)$, Mem. Amer. Math. Soc. 213, no. 1000.

[KØ] T. Kobayashi, B. Ørsted, Analysis on the minimal representation of $O(p, q) I, I I$, III, Adv. Math. 180 (2003), no. 2, 486-512, 513-550, 551-595.

[St] R. S. Strichartz, Harmonic analysis on hyperboloids, J. Functional Analysis 12 (1973), 341-383.

Department of Mathematics, Yale University, P.O. Box 208283, New Haven, CT 06520-8283

Department of Mathematics, Indiana University, Rawles Hall, 831 East 3rd St, Bloomington, IN 47405

Corresponding author's e-mail: mlibine@indiana.edu 